\title{
Ricci Solitons on Multiply Warped Product Manifolds
}

\author{
Dilek Açıkgöz Kaya* and Leyla Onat \\ (Communicated by Henrique F. De Lima)
}

\begin{abstract}
In this paper, we study Ricci solitons with the structure of multiply warped product manifolds. We also study Ricci solitons equipped with the concurrent vector fields on multiply warped product manifolds.
\end{abstract}

Keywords: Ricci soliton; gradient Ricci soliton; warped product; multiply warped product.

AMS Subject Classification (2020): 53C25

\section{Introduction}

Einstein metrics have an important place in the frontier between mathematics and physics. In the last few decades, many researchers focused to this special class of metrics as well as some similar structures that are called Einstein type metrics [1]. One of the most famous among these which is introduced by Hamilton[9] is the concept of Ricci solitons $[3,7,12,13,14,15]$. A Ricci soliton is a Riemannian metric together with a vector field $X$ and a scalar $\lambda$ which satisfies

$$
\operatorname{Ric}+\frac{1}{2} \mathcal{L}_{X} g=\lambda g
$$

$(M, g, X, \lambda)$ is called shrinking, steady and expanding when $\lambda>0, \lambda=0$ and $\lambda<0$, respectively. When $X$ is gradient of a function $f,(M, g, \nabla f, \lambda)$ is called a gradient Ricci soliton and equation (1.1) becomes

$$
\text { Ric }+\nabla^{2} f=\lambda g
$$

where $\nabla^{2} f$ stands for the Hessian of $f$.

Lately, there is a special interest on the relation between warped product manifolds (introduced in [2]) and Ricci solitons. In $[6,8,16]$, the authors studied gradient Ricci solitons with the structure of warped products and considered the potential function of the gradient Ricci soliton so that the lift of a function defined on base or fiber. In [10], this idea is extended to gradient Ricci solitons with the structure of multiply warped product manifolds.

In [11], the authors investigated the inheritance properties of Ricci soliton warped product manifolds by their factor manifolds. Motivating from this, we consider Ricci solitons with the structure of multiply warped product manifolds and reach some hereditary properties to its factors.

\section{Preliminaries}

In this section, we give a brief summary of multiply warped products defined in [17].

Definition 2.1 ([17]). Let $\left(B, g_{B}\right)$ and $\left(F_{i}, g_{F_{i}}\right)$ be $r$ and $s_{i}$ dimensional Riemannian manifolds where $i \in$ $\{1,2, \cdots, m\}$ and also $M=B \times F_{1} \times F_{2} \times \cdots \times F_{m}$ be an $n$-dimensional Riemannian manifold, 
where $n=r+\sum_{i=1}^{m} s_{i}$. Let $b_{i}: B \longrightarrow \mathbb{R}^{+}$be smooth functions for $i \in\{1,2, \cdots, m\}$. The multiply warped product is the product manifold $B \times_{b_{1}} F_{1} \times_{b_{2}} F_{2} \times_{b_{3}} \cdots \times_{b_{m}} F_{m}$ furnished with the metric tensor $g=g_{B} \oplus$ $b_{1}^{2} g_{F_{1}} \oplus \cdots \oplus b_{m}^{2} g_{F_{m}}$ defined by

$$
g=\pi^{*}\left(g_{B}\right) \oplus\left(b_{1} \circ \pi\right)^{2} \sigma_{1}^{*}\left(g_{F_{1}}\right) \oplus \cdots \oplus\left(b_{m} \circ \pi\right)^{2} \sigma_{m}^{*}\left(g_{F_{m}}\right)
$$

where $\pi$ and $\sigma_{i}$ are the natural projections on $B$ and $F_{i}$, respectively. The functions $b_{i}$ are called the warping functions for $i \in\{1,2, \cdots, m\}$. If $m=1$, then we obtain a singly warped product. If all $b_{i} \equiv 1$, then we have a product manifold.

We denote $\nabla,{ }^{B} \nabla$ and ${ }^{F_{i}} \nabla$; Ric, ${ }^{B}$ Ric and ${ }^{F_{i}}$ Ric the Levi-Civita connections and Ricci curvatures of the $M, B$ and $F_{i}$, respectively.

The lift of $X$ to $\mathrm{M}$ is the unique element of $\mathfrak{X}(M)$ that is $\pi$-related to $X$ and $\sigma_{i}$-related to zero vector field on $B$. Similarly, $V_{i} \in \mathfrak{X}\left(F_{i}\right)$ can be lifted to $M$ and the set of all such lifts is denoted by $\mathfrak{L}\left(F_{i}\right)$. We will use the same notation for a vector field and its lift.

Now, we recall the following formulas for multiply warped products.

Lemma 2.1 ([5]). $M=B^{r} \times_{b_{1}} F_{1}^{s_{1}} \times_{b_{2}} F_{2}^{s_{2}} \times_{b_{3}} \cdots \times_{b_{m}} F_{m}^{s_{m}}$ be a multiply warped product manifold. For $X, Y \in \mathfrak{L}(B)$, $V \in \mathfrak{L}\left(F_{i}\right)$ and $W \in \mathfrak{L}\left(F_{j}\right)$,

(i) $\nabla_{X} Y$ is the lift of ${ }^{B} \nabla_{X} Y$ on $B$,

(ii) $\nabla_{X} V=\nabla_{V} X=\frac{X\left(b_{i}\right)}{b_{i}} V$,

(iii) $\nabla_{V} W= \begin{cases}0 & \text { if } i \neq j, \\ F_{i} \nabla_{V} W-\left(\frac{g(V, W)}{b_{i}}\right) \operatorname{grad}_{B}\left(b_{i}\right) & \text { if } i=j .\end{cases}$

Lemma 2.2 ([5]). $M=B^{r} \times_{b_{1}} F_{1}^{s_{1}} \times_{b_{2}} F_{2}^{s_{2}} \times_{b_{3}} \cdots \times_{b_{m}} F_{m}^{s_{m}}$ be a multiply warped product manifold. For $X, Y \in \mathfrak{L}(B)$, $V \in \mathfrak{L}\left(F_{i}\right)$ and $W \in \mathfrak{L}\left(F_{j}\right)$,

(i) $\operatorname{Ric}(X, Y)={ }^{B} \operatorname{Ric}(X, Y)-\sum_{i=1}^{m} \frac{s_{i}}{b_{i}} \operatorname{Hess}_{B}^{b_{i}}(X, Y)$,

(ii) $\operatorname{Ric}(X, V)=0$,

(iii) For $i \neq j, \operatorname{Ric}(X, V)=0$,

(iv) For $i=j$,

$$
\begin{aligned}
\operatorname{Ric}(V, W)={ }^{F_{i}} \operatorname{Ric}(V, W)- & {\left[\frac{\Delta_{B} b_{i}}{b_{i}}+\left(s_{i}-1\right) \frac{\left|\operatorname{grad}_{B} b_{i}\right|^{2}}{b_{i}^{2}}\right.} \\
& \left.+\sum_{\substack{k=1 \\
k \neq i}}^{m} s_{k} \frac{g_{B}\left(\operatorname{grad}_{B} b_{i}, \operatorname{grad}_{B} b_{k}\right)}{b_{i} b_{k}}\right] g(V, W) .
\end{aligned}
$$

Throughout this paper, we will consider multiply warped product manifold with same warping functions, i.e., $b_{i}=b: B \longrightarrow \mathbb{R}$ for $i \in\{1,2, \cdots, m\}$. Emanating from this point, we conclude the following proposition.

Proposition 2.1. Let $M=B^{r} \times_{b} F_{1}^{s_{1}} \times_{b} F_{2}^{s_{2}} \times_{b} \cdots \times_{b} F_{m}^{s_{m}}$ be a multiply warped product manifold endowed with the metric $g=g_{B}+b^{2} \sum_{i=1}^{m} g_{F_{i}}$ and $\bar{X}=X+V_{i}$ be a vector field on $M$ such that $X \in \mathfrak{L}(B)$ and $V_{i} \in \mathfrak{L}\left(F_{i}\right)$. Then,

$$
\mathcal{L}_{\bar{X}} g=\mathcal{L}_{X}^{B} g_{B}+b^{2} \sum_{i=1}^{m} \mathcal{L}_{V_{i}}^{F_{i}} g_{F_{i}}+2 b X(b) \sum_{i=1}^{m} g_{F_{i}} .
$$


Proof. Let $\bar{X}=X+V_{i}, \bar{Y}=Y+W_{i}, \bar{Z}=Z+U_{i}$ be vector fields on $M$ such that $X, Y, Z \in \mathfrak{L}(B)$ and $V_{i}, W_{i}, U_{i} \in$ $\mathfrak{L}\left(F_{i}\right)$ for $i \in\{1,2, \cdots, m\}$.

$$
\begin{aligned}
\mathcal{L}_{\bar{X}} g(\bar{Y}, \bar{Z})= & g\left(\nabla_{\bar{Y}} \bar{X}, \bar{Z}\right)+g\left(\bar{Y}, \nabla_{\bar{Z}} \bar{X}\right) \\
= & g\left(\nabla_{\left(Y+W_{i}\right)}\left(X+V_{i}\right), Z+U_{i}\right)+g\left(Y+W_{i}, \nabla_{\left(Z+U_{i}\right)}\left(X+V_{i}\right)\right) \\
= & g\left(\nabla_{Y} X, Z\right)+g\left(\nabla_{W_{i}} V_{i}, Z\right)+g\left(\nabla_{Y} V_{i}, U_{i}\right)+g\left(\nabla_{W_{i}} X, U_{i}\right)+g\left(\nabla_{W_{i}} V_{i}, U_{i}\right) \\
& +g\left(Y, \nabla_{Z} X\right)+g\left(Y, \nabla_{U_{i}} V_{i}\right)+g\left(W_{i}, \nabla_{Z} V_{i}\right)+g\left(W_{i}, \nabla_{U_{i}} X\right)+g\left(W_{i}, \nabla_{U_{i}} V_{i}\right) \\
= & g_{B}\left(\nabla_{Y} X, Z\right)+g\left(-\frac{g\left(W_{i}, V_{i}\right)}{b} \nabla b, Z\right)+g\left(\frac{Y(b)}{b} V_{i}, U_{i}\right)+g\left(\frac{X(b)}{b} W_{i}, U_{i}\right) \\
& +b^{2} g_{F_{i}}\left(\nabla_{W_{i}} V_{i}, U_{i}\right)+g_{B}\left(Y, \nabla_{Z} X\right)+g\left(Y,-\frac{g\left(U_{i}, V_{i}\right)}{b} \nabla b\right)+g\left(W_{i}, \frac{Z(b)}{b} V_{i}\right) \\
& +g\left(W_{i}, \frac{X(b)}{b} U_{i}\right)+b^{2} g_{F_{i}}\left(W_{i}, \nabla_{U_{i}} V_{i}\right) \\
= & g_{B}\left(\nabla_{Y} X, Z\right)+g_{B}\left(Y, \nabla_{Z} X\right)+b^{2} g_{F_{i}}\left(\nabla_{W_{i}} V_{i}, U_{i}\right)+b^{2} g_{F_{i}}\left(W_{i}, \nabla_{U_{i}} V_{i}\right) \\
& +2 b X(b) g_{F_{i}}\left(W_{i}, U_{i}\right)
\end{aligned}
$$

which implies the equation (2.1).

\section{Main Results}

In this section, we examine the inheritance properties of Ricci solitons and gradient Ricci solitons on multiply warped product manifolds in order to characterize their factor manifolds.

Theorem 3.1. Let $M=B^{r} \times_{b} F_{1}^{s_{1}} \times_{b} F_{2}^{s_{2}} \times_{b} \cdots \times_{b} F_{m}^{s_{m}}$ be a multiply warped product manifold endowed with the metric $g=g_{B}+b^{2} \sum_{i=1}^{m} g_{F_{i}}$. If $(M, g, \bar{X}, \lambda)$ is a Ricci soliton with $\bar{X}=X+V_{i}$ where $X \in \mathfrak{L}(B)$ and $V_{i} \in \mathfrak{L}\left(F_{i}\right)$, then the base manifold $\left(B, g_{B}, X-\left(\sum_{i=1}^{m} s_{i}\right) \nabla(\ln b), \lambda\right)$ is a Ricci soliton and all of the fiber manifolds $\left(F_{i}, g_{F_{i}}, b^{2} V_{i}, \lambda b^{2}\right)$ are Ricci solitons when $b$ is constant.

Proof. Let $M=B^{r} \times_{b} F_{1}^{s_{1}} \times_{b} F_{2}^{s_{2}} \times_{b} \cdots \times_{b} F_{m}^{s_{m}}$ be a Ricci soliton. Then for all $\bar{Y}=Y+W_{i}, \bar{Z}=Z+U_{i} \in \mathfrak{X}(M)$, the equation

$$
\operatorname{Ric}(\bar{Y}, \bar{Z})+\frac{1}{2} \mathcal{L}_{\bar{X}} g(\bar{Y}, \bar{Z})=\lambda g(\bar{Y}, \bar{Z})
$$

is satisfied. From Lemma 2.2, we have

$$
\begin{aligned}
\operatorname{Ric}(\bar{Y}, \bar{Z})= & { }^{B} \operatorname{Ric}(Y, Z)-\frac{\sum_{i=1}^{m} s_{i}}{b} \operatorname{Hess}_{B}^{b}(Y, Z)+\sum_{i=1}^{m}{ }^{m} \operatorname{Ric}\left(W_{i}, U_{i}\right) \\
& -\left[b \Delta b+\left(\sum_{i=1}^{m} s_{i}-1\right)|\nabla b|^{2}\right] \sum_{i=1}^{m} g_{F_{i}}\left(W_{i}, U_{i}\right) .
\end{aligned}
$$

Using this equation and Proposition 2.1 in equation (3.1) we get

$$
\begin{gathered}
{ }^{B} \operatorname{Ric}(Y, Z)-\frac{\sum_{i=1}^{m} s_{i}}{b} \operatorname{Hess}_{B}^{b}(Y, Z)+\sum_{i=1}^{m}{ }^{F_{i}} \operatorname{Ric}\left(W_{i}, U_{i}\right)-\left[b \Delta b+\left(\sum_{i=1}^{m} s_{i}-1\right)|\nabla b|^{2}\right] \sum_{i=1}^{m} g_{F_{i}}\left(W_{i}, U_{i}\right) \\
+\frac{1}{2} \mathcal{L}_{X}^{B} g_{B}(Y, Z)+\frac{1}{2} b^{2} \sum_{i=1}^{m} \mathcal{L}_{V_{i}}^{F_{i}} g_{F_{i}}\left(W_{i}, U_{i}\right)+b X(b) \sum_{i=1}^{m} g_{F_{i}}\left(W_{i}, U_{i}\right) \\
=\lambda g_{B}(Y, Z)+\lambda b^{2} \sum_{i=1}^{m} g_{F_{i}}\left(W_{i}, U_{i}\right)
\end{gathered}
$$


So, we may conclude

$$
{ }^{B} \operatorname{Ric}(Y, Z)-\frac{\sum_{i=1}^{m} s_{i}}{b} \operatorname{Hess}_{B}^{b}(Y, Z)+\frac{1}{2} \mathcal{L}_{X}^{B} g_{B}(Y, Z)=\lambda g_{B}(Y, Z)
$$

and

$$
\begin{aligned}
& \sum_{i=1}^{m}{ }^{F_{i}} \operatorname{Ric}\left(W_{i}, U_{i}\right)-\left[b \Delta b+\left(\sum_{i=1}^{m} s_{i}-1\right)|\nabla b|^{2}\right] \sum_{i=1}^{m} g_{F_{i}}\left(W_{i}, U_{i}\right) \\
& +\frac{1}{2} b^{2} \sum_{i=1}^{m} \mathcal{L}_{V_{i}}^{F_{i}} g_{F_{i}}\left(W_{i}, U_{i}\right)+b X(b) \sum_{i=1}^{m} g_{F_{i}}\left(W_{i}, U_{i}\right)=\lambda b^{2} \sum_{i=1}^{m} g_{F_{i}}\left(W_{i}, U_{i}\right)
\end{aligned}
$$

The equation (3.4) implies that for each $i \in\{1,2, \cdots, m\}$, the fiber $F_{i}$ is a Ricci soliton with potential field $b^{2} V_{i}$ and constant $\lambda b^{2}$ when $b$ is constant.

$$
\frac{1}{2} \mathcal{L}_{X}^{B} g_{B}(Y, Z)-\frac{\sum_{i=1}^{m} s_{i}}{b} \operatorname{Hess}_{B}^{b}(Y, Z)=\frac{1}{2} \mathcal{L}_{X-\sum_{i=1}^{B} s_{i} \nabla(\ln b)} g_{B}(Y, Z),
$$

in equation (3.3) gives us

$$
{ }^{B} \operatorname{Ric}+\frac{1}{2} \mathcal{L}_{X-\sum_{i=1}^{B} s_{i} \nabla(\ln b)} g_{B}=\lambda g_{B}
$$

which means the base $B$ is also a Ricci soliton.

Using Theorem 3.1 we can state the following corollary.

Corollary 3.1. Let $M=B^{r} \times_{b} F_{1}^{s_{1}} \times_{b} F_{2}^{s_{2}} \times_{b} \cdots \times_{b} F_{m}^{s_{m}}$ be a multiply warped product manifold endowed with the metric $g=g_{B}+b^{2} \sum_{i=1}^{m} g_{F_{i}}$. If $(M, g, \bar{X}, \lambda)$ is a Ricci soliton and $X$ is a Killing vector field on $B$, then the base $\left(B, g_{B},-\left(\sum_{i=1}^{m} s_{i}\right) \nabla(\ln b), \lambda\right)$ is a gradient Ricci soliton.

Remark 3.1. Let $M=B^{r} \times_{b} F_{1}^{s_{1}} \times_{b} F_{2}^{s_{2}} \times_{b} \cdots \times_{b} F_{m}^{s_{m}}$ be a multiply warped product manifold endowed with the metric $g=g_{B}+b^{2} \sum_{i=1}^{m} g_{F_{i}}$. Assume that $(M, g, \bar{X}, \lambda)$ is a Ricci soliton. Then $(M, g)$ is Einstein if one of the following conditions holds:

(i) $\bar{X}=X$ and $X$ is a Killing vector field on $B$.

(ii) $\bar{X}=\sum_{i=1}^{m} V_{i}$ and each $V_{i}$ is a Killing vector field on $F_{i}$ for $i \in\{1, \cdots, m\}$.

(iii) $X$ and each $V_{i}$ are Killing vector fields on $B$ and $F_{i}$ for $i \in\{1, \cdots, m\}$, respectively and $X(b)=0$.

In the following theorem, we consider Riemannian manifolds with certain properties so that their multiply warped product manifold has the structure of Ricci soliton.

Theorem 3.2. Let $\left(B, g_{B}, X, \lambda\right)$ be a Ricci soliton and $\left(F_{i}^{s_{i}}, g_{F_{i}}\right)$ be an Einstein manifold with factor $\mu_{i}$ for each $i \in\{1, \cdots, m\}$. Then

$\left(M=B^{r} \times_{b} F_{1}^{s_{1}} \times_{b} \cdots \times_{b} F_{m}^{s_{m}}, g=g_{B}+b^{2} \sum_{i=1}^{m} g_{F_{i}}, \bar{X}, \lambda\right)$ is a Ricci soliton if the following conditions hold

(i) For each $i \in\{1, \cdots, m\}, V_{i}$ is conformal with factor $2 \rho_{i}$,

(ii) $\operatorname{Hess}_{B}^{b}=0$,

(iii) $\left(\lambda-\rho_{i}\right) b^{2}=b X(b)+\mu_{i}-\left(\left(\sum_{i=1}^{m} s_{i}\right)-1\right)|\nabla b|^{2}$ for each $i \in\{1, \cdots, m\}$. 
Proof. Let $\left(B, g_{B}, X, \lambda\right)$ be a Ricci soliton and $\left(F_{i}^{s_{i}}, g_{F_{i}}\right)$ be an Einstein manifold with factor $\mu_{i}$ for each $i \in\{1, \cdots, m\}$. Then we have

$$
{ }^{B} \operatorname{Ric}+\frac{1}{2} \mathcal{L}_{X}^{B} g_{B}=\lambda g_{B}
$$

and ${ }^{F_{i}}$ Ric $=\mu_{i} g_{F_{i}}$ for each $i \in\{1, \cdots, m\}$. Using the equation (3.2) and the conditions in the hypothesis, we arrive

$$
\begin{aligned}
\operatorname{Ric}+\frac{1}{2} \mathcal{L}_{\bar{X}} g= & \lambda g_{B}+\sum_{i=1}^{m} \mu_{i} g_{F_{i}}-\left[\left(\left(\sum_{i=1}^{m} s_{i}\right)-1\right)|\nabla b|^{2}\right] \sum_{i=1}^{m} g_{F_{i}} \\
& +b^{2} \sum_{i=1}^{m} \rho_{i} g_{F_{i}}+b X(b) \sum_{i=1}^{m} g_{F_{i}} \\
= & \lambda g_{B}+b^{2} \sum_{i=1}^{m} \rho_{i} g_{F_{i}}+\sum_{i=1}^{m}\left[\mu_{i}-\left(\left(\sum_{j=1}^{m} s_{j}\right)-1\right)|\nabla b|^{2}+b X(b)\right] g_{F_{i}} \\
= & \lambda g_{B}+b^{2} \sum_{i=1}^{m} \rho_{i} g_{F_{i}}+\sum_{i=1}^{m}\left(\lambda-\rho_{i}\right) b^{2} g_{F_{i}} \\
= & \lambda g
\end{aligned}
$$

which implies $(M, g, \bar{X}, \lambda)$ is a Ricci soliton.

Now, we give the inheritance properties of gradient Ricci soliton multiply warped product.

Theorem 3.3. Let $M=B \times_{b} F_{1} \times_{b} F_{2} \times_{b} \cdots \times_{b} F_{m}$ be a multiply warped product such that $(M, g, \phi, \lambda)$ is a gradient Ricci soliton. Then,

(i) $\left(B, g_{B}, u, \lambda\right)$ is a gradient Ricci soliton with $u=\phi_{1}-\sum s_{i} \ln b$ and $\phi_{1}=\phi$ at some fixed point of $F_{1} \times F_{2} \times \cdots \times F_{m}$

(ii) $\left(F_{i}, g_{F_{i}}, u_{i}, \lambda b^{2}\right)$ is a gradient Ricci soliton with $u_{i}=\phi$ at some fixed point of $B$ if $b$ is constant.

Proof. Assume that $(M, g, \phi, \lambda)$ is a gradient Ricci soliton. For $\bar{Y}, \bar{Z} \in \mathfrak{X}(M)$,

$$
\operatorname{Ric}(\bar{Y}, \bar{Z})+\nabla^{2} \phi(\bar{Y}, \bar{Z})=\lambda g(\bar{Y}, \bar{Z})
$$

is satisfied. Let $\bar{Y}=Y, \bar{Z}=Z$, then equation (3.5) becomes

$$
\begin{gathered}
{ }^{B} \operatorname{Ric}(Y, Z)-\sum_{i=1}^{m} \frac{s_{i}}{b} \operatorname{Hess}_{B}^{b}(Y, Z)+\operatorname{Hess}_{B}^{\phi_{1}}(Y, Z)=\lambda g_{B}(Y, Z) \\
{ }^{B} \operatorname{Ric}(Y, Z)+\operatorname{Hess}_{B}^{u}(Y, Z)=\lambda g_{B}(Y, Z)
\end{gathered}
$$

where $u=\phi_{1}-\sum s_{i} \ln b$ and $\phi_{1}=\phi$ at some fixed point of $F_{1} \times F_{2} \times \cdots \times F_{m}$. Hence $B$ is a gradient Ricci soliton. Using the same pattern, (ii) can be verified.

\section{Multiply Warped Product Ricci Solitons with Concurrent Vector Fields}

In this section, we firstly recall the definition of a concurrent vector field on a Riemannian manifold, i.e., $X \in \mathfrak{X}(M)$ is said to be concurrent if it satisfies

$$
\nabla_{Z} X=X
$$

for any vector field $Z \in \mathfrak{X}(M)[4]$.

The next result gives the necessary and sufficient conditions for components of concurrent vector fields on a multiply warped product manifold. 
Proposition 4.1. Let $\bar{X}=X+V_{i}$ be a vector field on $M^{n}=B^{r} \times_{b} F_{1}^{s_{1}} \times_{b} F_{2}^{s_{2}} \times_{b} \cdots \times_{b} F_{m}^{s_{m}}$ where $X \in \mathfrak{L}(B)$ and $V_{i} \in \mathfrak{L}\left(F_{i}\right) . \bar{X}$ is a concurrent vector field on $M$ if and only if $X$ is a concurrent vector field on $B$ and one of the following conditions holds

(i) $V_{i}$ is a concurrent vector field on $F_{i}$ and $b$ is constant,

(ii) $V_{i}=0$ and $X(b)=b$.

Proof. Suppose that $\bar{X}$ is a concurrent vector field on $M$ and $\left\{\partial_{i}\right\}_{i=1}^{n}$ be a basis for $\mathfrak{X}(M)$. Then, for $1 \leq j \leq r$,

$$
\partial_{j}=\nabla_{\partial_{j}} \bar{X}=\nabla_{\partial_{j}}\left(X+V_{i}\right)=\nabla_{\partial_{j}}^{B} X+\sum_{i=1}^{m} \frac{\partial_{j}(b)}{b} V_{i} .
$$

By comparing the tangential and normal parts of the (4.1), we can conclude

$$
\nabla_{\partial_{j}}^{B} X=\partial_{j}
$$

and

$$
0=\frac{\partial_{j}(b)}{b} \sum_{i=1}^{m} V_{i}
$$

so that $X$ is a concurrent vector field on $B$ and $\frac{\partial_{j}(b)}{b}=0$ or $\sum_{i=1}^{m} V_{i}=0$.

Case 1: It is clear that $\frac{\partial_{j}(b)}{b}=0$ for $1 \leq j \leq r$ implies $b$ is constant. When $j \in\left\{r+1, r+2, \cdots, r+s_{1}, r+s_{1}+\right.$ $1, \cdots, n\}$, we get

$$
\partial_{j}=\nabla_{\partial_{j}} \bar{X}=\frac{X(b)}{b} \partial_{j}+\sum_{i=1}^{m} \nabla_{\partial_{j}}^{F_{i}} V_{i}-b \sum_{i=1}^{m} g_{F_{i}}\left(\partial_{j}, V_{i}\right) \nabla b .
$$

Since $b$ is constant, we have $\nabla_{\partial_{j}}^{F_{i}} V_{i}=\partial_{j}$, i.e., $V_{i}$ is concurrent vector field on $F_{i}$ for $1 \leq i \leq m$.

Case 2: Assuming $\sum_{i=1}^{m} V_{i}=0$ yields

$$
\partial_{j}=\nabla_{\partial_{j}} \bar{X}=\frac{X(b)}{b} \partial_{j}
$$

Therefore, we have $\frac{X(b)}{b}=1$, i.e., $(i i)$ holds.

The converse can be shown by direct calculation.

Using the above proposition, we reach the following theorem.

Theorem 4.1. Let $\bar{X}=X+V_{i}$ be a concurrent vector field on $M=B \times_{b} F_{1} \times_{b} F_{2} \times_{b} \cdots \times_{b} F_{m}$ where $X \in \mathfrak{L}(B)$ and $V_{i} \in \mathfrak{L}\left(F_{i}\right)$ such that $(M, g, \bar{X}, \lambda)$ is a Ricci soliton. If $V_{i} \neq 0$, then $M, B$ and $F_{i}$ are Ricci flat, gradient Ricci soliton with $\lambda=1$.

Proof. Assume that $(M, g, \bar{X}, \lambda)$ is a Ricci soliton and $\bar{X}=X+V_{i}$ be a concurrent vector field on $M$. For $\bar{Y}, \bar{Z} \in \mathfrak{X}(M)$, we have

$$
\mathcal{L}_{\bar{X}} g(\bar{Y}, \bar{Z})=g\left(\nabla_{\bar{Y}} \bar{X}, \bar{Z}\right)+g\left(\bar{Y}, \nabla_{\bar{Z}} \bar{X}\right)=g(\bar{Y}, \bar{Z})+g(\bar{Y}, \bar{Z})=2 g(\bar{Y}, \bar{Z}) .
$$

Thus, we get

$$
\operatorname{Ric}(\bar{Y}, \bar{Z})=(\lambda-1) g(\bar{Y}, \bar{Z}) .
$$

In particular, for $\bar{Y}=\sum_{i=1}^{m} W_{i}, \bar{Z}=\sum_{i=1}^{m} U_{i}$ we have

$$
\begin{aligned}
& \operatorname{Ric}(\bar{Y}, \bar{Z})=\sum_{i=1}^{m} \operatorname{Ric}\left(W_{i}, U_{i}\right) \\
& =\sum_{i=1}^{m}{ }^{F_{i}} \operatorname{Ric}\left(W_{i}, U_{i}\right)-\left[b \Delta b+\left(\sum_{i=1}^{m} s_{i}-1\right)|\nabla b|^{2}\right] \sum_{i=1}^{m} g_{F_{i}}\left(W_{i}, U_{i}\right) .
\end{aligned}
$$


Combining equations (4.2) and (4.3), we have

$$
(\lambda-1) b^{2} \sum_{i=1}^{m} g_{F_{i}}\left(W_{i}, U_{i}\right)=\sum_{i=1}^{m}{ }^{F_{i}} \operatorname{Ric}\left(W_{i}, U_{i}\right)-\left[b \Delta b+\left(\sum_{i=1}^{m} s_{i}-1\right)|\nabla b|^{2}\right] \sum_{i=1}^{m} g_{F_{i}}\left(W_{i}, U_{i}\right) .
$$

From $(i)$ of Proposition 4.1, we know that $V_{i}$ is concurrent for each $i \in\{1, \cdots, m\}$ and $b=c$ for some constant $c$. Hence, the above equation becomes

$$
\sum_{i=1}^{m}{ }^{F_{i}} \operatorname{Ric}\left(W_{i}, U_{i}\right)=\sum_{i=1}^{m}(\lambda-1) c^{2} g_{F_{i}}\left(W_{i}, U_{i}\right)
$$

i.e., $F_{i}$ is Einstein with factor $\mu=(\lambda-1) c^{2}$. Since this equation is valid for any vector field in $\mathfrak{X}\left(F_{i}\right)$, we may write

$$
{ }^{F_{i}} \operatorname{Ric}\left(V_{i}, V_{i}\right)=(\lambda-1) c^{2}\left|V_{i}\right|_{F_{i}}^{2} .
$$

Let $\left\{V_{i}, e_{1}, \cdots, e_{s_{i}-1}\right\}$ be an orthogonal basis of $\mathfrak{X}\left(F_{i}\right)$. Then, Riemann curvature tensor is

$$
\begin{aligned}
{ }^{F_{i}} \mathrm{R}\left(V_{i}, e_{j}, V_{i}, e_{j}\right) & =g_{F_{i}}\left({ }_{i_{i}} \mathrm{R}\left(V_{i}, e_{j}\right) V_{i}, e_{j}\right) \\
& =g_{F_{i}}\left(\nabla_{V_{i}} \nabla_{e_{j}} V_{i}-\nabla_{e_{j}} \nabla_{V_{i}} V_{i}-\nabla_{\left[V_{i}, e_{j}\right]} V_{i}, e_{j}\right) \\
& =g_{F_{i}}\left(\nabla_{V_{i}} e_{j}-\nabla_{e_{j}} V_{i}-\left[V_{i}, e_{j}\right], e_{j}\right) \\
& =0
\end{aligned}
$$

which implies ${ }^{F_{i}} \operatorname{Ric}\left(V_{i}, V_{i}\right)=0$. From equation (4.5), we have $\lambda=1$. Moreover, $M$ and $F_{i}$ are Ricci flat from equations (4.2) and (4.4).

Now, suppose that $\bar{Y}=Y, \bar{Z}=Z$. Then

$$
\begin{aligned}
0=\operatorname{Ric}(\bar{Y}, \bar{Z}) & =\operatorname{Ric}(Y, Z) \\
& ={ }^{B} \operatorname{Ric}(Y, Z)-\sum_{i=1}^{m} \frac{s_{i}}{b} \operatorname{Hess}_{B}^{b}(Y, Z) \\
& ={ }^{B} \operatorname{Ric}(Y, Z)
\end{aligned}
$$

since $b$ is constant. Hence, $B$ is Ricci flat. It is clear that $M, B$ and $F_{i}$ are Ricci soliton with $\lambda=1$.

Since $g(\bar{Y}, \nabla \phi)=\bar{Y}(\phi)=g\left(\nabla_{\bar{Y}} \bar{X}, \bar{X}\right)=g(\bar{Y}, \bar{X})$ for $\phi=\frac{1}{2} g(\bar{X}, \bar{X}), M$ is a gradient Ricci soliton with potential function $\phi$. Similarly, one can show that $B$ and $F_{i}$ are gradient Ricci solitons with potential functions $u=$ $\frac{1}{2} g(X, X)$ and $u_{i}=\frac{1}{2} g\left(V_{i}, V_{i}\right)$.

\section{References}

[1] Besse, A.L.: Einstein Manifolds, Classics in Mathematics, Springer-Verlag, Berlin (2008).

[2] Bishop, R.L., O’Neill, B.: Manifolds of negative curvature. Trans. Amer. Math. Soc. 145, 1-49 (1969).

[3] Cao, H-D., Zhou,D.: On complete gradient shrinking Ricci solitons. Journal of Differential Geometry. 85, 175-186 (2010).

[4] Chen, B-Y.: Some results on concircular vector fields and their applications to Ricci solitons. Bull. Korean Math. Soc. 52 No. 5, 1535-1547 (2015).

[5] Dobarro, F., Ünal, B.: Curvature of multiply warped products. J. Geom. Phys. 55, 75-106 (2005).

[6] Feitosa, F.E.S.,Freitas Filho A.A., Gomes, J.N.V.: On the construction of gradient Ricci soliton warped product. Nonlinear Analysis 161, 30-43 (2017).

[7] Fernandez-Lopez, M., Garcia-Rio, E.: Rigidity of shrinking Ricci solitons. Mathematische Zeitschrift. 269, 461-466 (2011).

[8] Günsen, S., Onat, L. and Açıkgöz Kaya, D.: The warped product manifold as gradient Ricci soliton and relation to its components. C. R. Acad. Bulg. Sci. 72 (8), 1015-1023 (2019).

[9] Hamilton R.S.: Three-manifolds with positive Ricci curvature. J. Differential Geom. 17 (2), 255-306 (1982).

[10] Karaca, F. and Özgür, C.: Gradient Ricci Solitons on Multiply Warped Product Manifolds. Filomat. 32 (12), 4221-4228 (2018).

[11] Mantica, C.A., Shenawy, S., Ünal, B.: Ricci Solitons on Singly Warped Product Manifolds and Applications. Preprint arxiv:1508.02794v2 2019).

[12] Munteanu, O., Sesum, N.: On Gradient Ricci Solitons. Journal of Geometric Analysis. 23, $539-561$ (2013).

[13] Petersen, P., Wylie, W.:Rigidity of gradient Ricci solitons. Pacific Journal of Mathematics. 241, 329-345 (2009).

[14] Petersen, P., Wylie, W.: On gradient Ricci solitons with symmetry. Proc. Amer. Math. Soc. 137, 2085-2092 (2009).

[15] Petersen, P., Wylie, W.: On the classification of gradient Ricci solitons. Geom. Topol. 14, 2277-2300 (2010).

[16] Sousa, M.L., Pina, R.: Gradient Ricci Solitons with Structure of Warped Product. Results Math. 71, 825-840 (2017).

[17] Ünal, B.: Multiply warped products. J. Geom. Phys. 34 , 287-301 (2000). MR1762779 and DOI 10.1016/S0393-0440(99)00072-8 


\section{Affiliations}

DILEK AÇIKGÖZ KAYA

AdDress: Aydın Adnan Menderes University, Dept. of Mathematics, 09010, Aydın-Turkey. E-MAIL: dilek.acikgoz@adu.edu.tr

ORCID ID:0000-0003-1603-9658

LEYLA ONAT

AdDress: Aydın Adnan Menderes University, Dept. of Mathematics, 09010, Aydın-Turkey. E-MAIL: lonat@adu.edu.tr

ORCID ID:0000-0002-9926-1467 